\section{Bridging non-communicable disease burden research to clinical care: A rising tide or a tidal wave?}

To the Editor: There is an increased focus on non-communicable diseases (NCDs) in low- and middle-income countries. ${ }^{[1]}$ South Africa (SA)'s National Strategic Plan on HIV looks to leverage the successes of community-based HIV programmes to build an effective NCD continuum of care. ${ }^{[2,3]}$ In early 2015, our research team added evidence of the colliding burden of non-infectious and infectious diseases in rural KwaZulu-Natal, SA through an integrated homebased NCD and HIV testing and counselling (HTC) programme. ${ }^{[4]}$ As parallels and differences between HIV and NCD programmes are being investigated, significant questions arise: (i) What is our ethical obligation in HIV- and NCD-integrated screening projects to followup testing and care?; and (ii) Where will the large number of at-risk patients go and how will it affect the current HIV programmes?

The benefits of early, integrated screening are clear. ${ }^{[5]}$ Early case identification maximises the benefits of lifestyle and pharmacological interventions. At the community level, integrated programmes may destigmatise HIV and promote a culture of preventive health. Furthermore, disease burden research helps policy-makers to set priorities and allocate resources.

We are reminded of the criteria for a justifiable screening programme, ${ }^{[6,7]}$ specifically that $(i)$ the costs of the programme must justify the benefits; and (ii) resources to run the programme must be available. Primum non nocere (first, do no harm) is the basis of the ethical principle of non-maleficence. In our study, numerous pitfalls arose as we reviewed the timing of the NCD testing and counselling relative to HTC. How did the health worker set priorities during counselling? What would the impact of NCD counselling be if emphasised before HIV counselling in the case of a high-risk patient? At what threshold is there diminishing return in broadening our counselling? At a systems level, we are challenged in our pursuit of distributive justice. Screening to identify high-volume, low-acuity NCD cases threatens to overburden an already challenged system. In isolation, linkage to care data is unlikely to capture the potential harms. Once enrolled, do NCD patients continue through the cascade of care? Does the influx of NCD patients draw resources and human capital away from higher-acuity HIV or tuberculosis (TB) cases? Is it ethical to focus on community-based NCD counselling and delay clinic referral that may potentially harm the system?

We propose the following principles to preserve the responsibility of guaranteeing benefit to the community: (i) a capacity assessment of the NCD care cascade should be conducted, aligned to the stability of coexisting HIV and TB programmes; (ii) the screening programme should be coupled with interventions to minimise the overburdening of clinics, including community-based strategies for lifestyle modifications and support groups, and potentially to initiate therapy with medications, which raises the question of funding, as US federal funds restrict ancillary care or capacity building (outside the scope of this letter) ${ }^{[8]}$ (iii) integrated screening programmes should support a steady rise rather than a flood of new patients; and (iv) the need to strengthen impact evaluations to provide feedback and ensure that integration brings more benefit than harm to the systems on which we build.

\section{Golovaty}

Department of Medicine, University of Washington, Seattle, WA, USA ilyamg@uw.edu

\section{A van Heerden, $Z$ Essack, $H$ van Rooyen}

Human Sciences Research Council, Pretoria, South Africa

\section{R Barnabas}

Department of Medicine, and Departments of Global Health and Epidemiology, University of Washington, Seattle, WA; and Vaccine and Infectious Diseases Division, Fred Hutchinson Cancer Research Center, Seattle, WA, USA

1. Lim SS, Vos T, Flaxman AD, et al. A comparative risk assessment of burden of disease and injur attributable to 67 risk factors and risk factor clusters in 21 regions, 1990 - 2010: A systematic analys for the Global Burden of Disease Study 2010. Lancet 2012;380(9859):2224-2260. http://dx.doi.org/ 10.1016/S0140-6736(12)61766-8

2. United Nations Programme on HIV/AIDS. Global AIDS response progress report. 2012. http://www. unaids.org/sites/default/files/country/documents//ce ZZ_Narrative_Report.pdf (accessed 20 April 2017).

3. Ministry of Health. Strategic plan for the prevention and control of non-communicable diseases $2013-17.2013$ http://www.hsrc.ac.za/uploads/pageContent/3893/NCDs\%20STRAT\%20PLAN\%20\%20CONTENT\%20 $8 \% 20$ april $\% 20$ proof.pdf (accessed 26 April 2017)

4. Van Heerden A, Barnabas R, Norris S, Micklesfield L. Integrating non-communicable disease (NCD) Van Heerden A, Barnabas R, Norris S, Micklesfield L. Integrating non-communicable disease (NCD)
screening and referral to care into home HIV testing and counseling in rural KwaZulu-Natal, South screening and referral to care into home HIV testing and counseling in rural KwaZulu-Natal, South
Africa: Burden of disease. International AIDS Conference, 18 - 22 July 2016, Durban, South Africa Africa: Burden of disease. International AIDS Conference, 18 - 22 July 2016, Du
http://repository.hsrc.ac.za/handle/20.500.11910/10129 (accessed 28 April 2017).

5. Checkley W, Ghannem H, Irazola V, et al. Management of NCD in low- and middle-income countries Glob Heart 2014;9(4):431-443. http://dx.doi.org/10.1016/j.gheart.2014.11.003

6. Sackett DL, Haynes RB, Guyatt GH, Tugwell P. Clinical Epidemiology: A Basic Science for Clinical Medicine. 2nd ed. Boston: Little, Brown, 1991:154-170.

Ewart R. Primum non nocere and the quality of evidence: Rethinking the ethics of screening. J Am Board Fam Pract 2000;13(3):188-196.

8. Philpott S, Slevin K, Shapiro K, Lori H. Impact of donor-imposed requirements and restrictions on standards of prevention and access to care and treatment in HIV prevention trials. Public Health Ethics 2010;3:220-228. http://dx.doi.org/10.1093/phe/phq027

S Afr Med J 2017;107(6):467. DOI:10.7196/SAMJ.2017.v107i6.12495 